\title{
On Scalar Weak Commutative Algebras
}

\author{
G.Gopalakrishnamoorthy ${ }^{1}$, S.Geetha ${ }^{2}$, S.Anitha ${ }^{3}$ \\ ${ }^{1}$ Principal, Sri krishnasamy Arts and Science College , Sattur - 626203, Tamilnadu. \\ ${ }^{2}$ Dept. of Mathematics, Pannai College of Engineering and Technology, Keelakkandani, Sivagangai - 630561. \\ ${ }^{3}$ Lecturer, Raja Doraisingam Government Arts College, Sivagangai - 630 561,Tamil Nadu.
}

Abstract: The concept of scalar commutativity defined in an algebra A over a ring $R$ is mixed with the concept of weak-commutativity defined in a Near-ring to coin the new concept of scalar weak commutativity in an algebra $A$ over a ring $R$ and many interesting results are obtained.

\section{Introduction}

Let $\mathrm{A}$ be an algebra (not necessarily associative) over a commutative ring R.A is called scalar commutative if for each $\mathrm{x}, \mathrm{y} \in \mathrm{A}$,there exists $\alpha \in \mathrm{R}$ depending on $\mathrm{x}, \mathrm{y}$ such that $\mathrm{xy}=\alpha \mathrm{yx}$.Rich[8] proved that if A is scalar commutative over a field F,then $\mathrm{A}$ is either commutative or anti-commutative. KOH,LUH and PUTCHA [6] proved that if A is scalar commutative with 1 and if $\mathrm{R}$ is a principal ideal domain ,then $\mathrm{A}$ is commutative. A near-ring $\mathrm{N}$ is said to be weak-commutative if $\mathrm{xyz}=\mathrm{xzy}$ for all $\mathrm{x}, \mathrm{y}, \mathrm{z} \in \mathrm{N}$ (Definition 9.4, p.289, Pliz[7]. In this paper we define scalar weak commutativity in an algebra A over a commutative ring $\mathrm{R}$ and prove many interesting results analogous to Rich and LUH.

\section{Preliminaries}

In this section we give some basic definitions and well known results which we use in the sequel.

2.1 Definition [ 7 ]:

Let $\mathrm{N}$ be a near-ring. $\mathrm{N}$ is said to be weak commutative if $\mathrm{xyz}=\mathrm{xzy}$ for all $\mathrm{x}, \mathrm{y}, \mathrm{z} \in \mathrm{N}$.

2.2 Definition:

Let $\mathrm{N}$ be a near-ring. $\mathrm{N}$ is said to be anti-weak commutative if $\mathrm{xyz}=-\mathrm{xzy}$ for all $\mathrm{x}, \mathrm{y}, \mathrm{z} \in \mathrm{N}$.

2.3 Definition [ 8 ]:

Let $\mathrm{A}$ be an algebra (not necessarily associative) over a commutative ring R.A is called scalar commutative if for each $\mathrm{x}, \mathrm{y} \in \mathrm{A}$,there exists $\alpha=\alpha(\mathrm{x}, \mathrm{y}) \in \mathrm{R}$ depending on $\mathrm{x}, \mathrm{y}$ such that $\mathrm{xy}=\alpha \mathrm{yx}$. A is called scalar anticommutative if $\mathrm{xy}=-\alpha \mathrm{yx}$.

\subsection{Lemma[5]:}

Let $N$ be a distributive near-ring.If $x y z= \pm$ xzy for all $x, y, z \in N$, then $N$ is either weak commutative or weak anti-commutative.

\subsection{Definition}

\section{Main Results}

Let $\mathrm{A}$ be an algebra (not necessarily associative) over a commutative ring R. A is called scalar weakcommutative if for every $\mathrm{x}, \mathrm{y}, \mathrm{z} \in \mathrm{A}$, there exists $\alpha=\alpha(\mathrm{x}, \mathrm{y}, \mathrm{z}) \in \mathrm{R}$ depending on $\mathrm{x}, \mathrm{y}, \mathrm{z}$ such that $\mathrm{xyz}=\alpha \mathrm{xzy}$. A is called scalar anti-weak commutative if $x y z=-\alpha x z y$.

\subsection{Theorem:}

Let A be an algebra ( not necessarily associative) over a field F.If A is scalar weak commutative,then A is either weak commutative or anti- weak commutative.

Proof:

Suppose $\mathrm{xyz}=\mathrm{xzy}$ for all $\mathrm{x}, \mathrm{y}, \mathrm{z} \in \mathrm{A}$, there is nothing to prove.

Suppose not we shall prove that $\mathrm{xyz}=-\mathrm{xzy}$ for all $\mathrm{x}, \mathrm{y}, \mathrm{z} \in \mathrm{A}$.

We shall first prove that,if $\mathrm{x}, \mathrm{y}, \mathrm{z} \in \mathrm{A}$ such that $\mathrm{xyz} \neq \mathrm{xzy}$, then $\mathrm{x} \mathrm{y}^{2}=\mathrm{x} \mathrm{z}^{2}=0$.

Let $\mathrm{x}, \mathrm{y}, \mathrm{z} \in \mathrm{A}$ such that $\mathrm{xyz} \neq \mathrm{xzy}$.

Since A is scalar weak commutative,there exists $\alpha=\alpha(\mathrm{x}, \mathrm{y}, \mathrm{z}) \in \mathrm{F}$ such that

$$
\mathrm{xyz}=\alpha \mathrm{xzy}
$$

Also there exists $\gamma=\gamma(\mathrm{x}, \mathrm{y}+\mathrm{z}, \mathrm{z}) \in \mathrm{F}$ such that

(1) $\quad-(2)$ gives

$$
\mathrm{x}(\mathrm{y}+\mathrm{z}) \mathrm{z}=\gamma \mathrm{xz}(\mathrm{y}+\mathrm{z})
$$

$$
\begin{aligned}
& \mathrm{xyz}-\mathrm{xyz}-\mathrm{xz}^{2}=\alpha \mathrm{xzy}-\gamma \mathrm{xzy}-\gamma \mathrm{xz}^{2} . \\
& \gamma \mathrm{xz}^{2}-\mathrm{xz}^{2}=(\alpha-\gamma) \mathrm{xzy} .
\end{aligned}
$$


$\mathrm{x} \mathrm{z}^{2}-\gamma \mathrm{x} \mathrm{z}^{2}=(\gamma-\alpha) \mathrm{xzy}$

Now, $x z y \neq 0$ for if $x z y=0$, then from(1), we get $x y z=0$ and so $x y z=x z y$;

contradicting our assumption that $\mathrm{xyz} \neq \mathrm{xzy}$.

Also $\gamma \neq 1$, for if $\gamma=1$, then from (3) we get

Then from (1) we get

$$
\alpha=\gamma=1
$$

Now from (3) we get

$\mathrm{xyz}=\mathrm{xzy}$, again contradicting assumption that $\mathrm{xyz} \neq \mathrm{xzy}$.

$$
\begin{gathered}
\mathrm{x} \mathrm{z}^{2}=\frac{\gamma-\alpha}{1-\gamma} \quad \mathrm{xzy} . \\
\text { i.e., } \quad \mathrm{x} \mathrm{z}^{2}=\beta \mathrm{xzy} \text { for some } \beta \in \mathrm{F} .
\end{gathered}
$$

Similarly $\mathrm{x} \mathrm{y}^{2}=\delta$ xzy for some $\delta \in \mathrm{F}$

Now corresponding to each choice of $\alpha_{1}, \alpha_{2}, \alpha_{3}, \alpha_{4}$ in F,there is an $\eta \in \mathrm{F}$ such that

$$
\begin{aligned}
& \mathrm{x}\left(\alpha_{1} \mathrm{y}+\alpha_{2} \mathrm{z}\right)\left(\alpha_{3} \mathrm{y}+\alpha_{4} \mathrm{z}\right)=\eta \mathrm{x}\left(\alpha_{3} \mathrm{y}+\alpha_{4} \mathrm{z}\right)\left(\alpha_{1} \mathrm{y}+\alpha_{2} \mathrm{z}\right) \\
& \mathrm{x}\left(\alpha_{1} \alpha_{3} \mathrm{y}^{2}+\alpha_{1} \alpha_{4} \mathrm{yz}+\alpha_{2} \alpha_{3} \mathrm{zy}+\alpha_{2} \alpha_{4} \mathrm{z}^{2}\right) \\
& =\eta \mathrm{x}\left(\alpha_{3} \alpha_{1} \mathrm{y}^{2}+\alpha_{3} \alpha_{2} \mathrm{yz}+\alpha_{4} \alpha_{1} \mathrm{zy}+\alpha_{4} \alpha_{2} \mathrm{z}^{2}\right) \\
& \alpha_{1} \alpha_{3} \mathrm{x} \mathrm{y}^{2}+\alpha_{1} \alpha_{4} \mathrm{xyz}+\alpha_{2} \alpha_{3} \mathrm{xzy}+\alpha_{2} \alpha_{4} \mathrm{xz}^{2} \\
& =\eta\left(\alpha_{3} \alpha_{1} \mathrm{xy}^{2}+\alpha_{3} \alpha 1 \alpha_{2} \mathrm{xyz}+\alpha_{4} \alpha_{1} \mathrm{xzy}+\alpha_{4} \alpha_{2} \mathrm{xz}^{2}\right) \\
& \text { Using (4) and (5) we get, } \\
& \alpha_{1} \alpha_{3} \delta \mathrm{xzy}+\alpha_{1} \alpha_{4} \mathrm{x} \mathrm{yz}+\alpha_{2} \alpha_{3} \mathrm{xzy}+\alpha_{2} \alpha_{4} \beta \mathrm{xzy} \\
& =\eta\left(\alpha_{3} \alpha_{1} \delta \mathrm{xzy}+\alpha_{3} \alpha_{2} \mathrm{xyz}+\alpha_{4} \alpha_{1} \mathrm{xzy}+\alpha_{4} \alpha_{2} \beta \mathrm{xzy}\right) .
\end{aligned}
$$

Using (1) we get,

$$
\begin{gathered}
\alpha_{1} \alpha_{3} \delta \alpha^{-1} \mathrm{xyz}+\alpha_{1} \alpha_{4} \mathrm{xyz}+\alpha_{2} \alpha_{3} \alpha^{-1} \mathrm{xyz}+\alpha_{2} \alpha_{4} \beta \alpha^{-1} \mathrm{xyz} \\
=\eta\left(\alpha_{3} \alpha_{1} \delta \mathrm{xzy}+\alpha_{3} \alpha_{2} \alpha \mathrm{xzy}+\alpha_{4} \alpha_{1} \mathrm{x} \mathrm{zy}+\alpha_{4} \alpha_{2} \beta \mathrm{xzy}\right) \\
\left(\alpha_{1} \alpha_{3} \delta \alpha^{-1}+\alpha_{1} \alpha_{4}+\alpha_{2} \alpha_{3} \alpha^{-1}+\alpha_{2} \alpha_{4} \beta \alpha^{-1}\right) \mathrm{xyz} \\
=\eta\left(\alpha_{3} \alpha_{1} \delta+\alpha_{3} \alpha_{2} \alpha+\alpha_{4} \alpha_{1}+\alpha_{4} \alpha_{2}\right) \mathrm{xzy}
\end{gathered}
$$

If in (7), we choose $\alpha_{2}=0, \alpha_{3}=\alpha_{1}=1, \alpha_{4}=-\delta$, the right hand side of (7) is zero

Whereas the left hand side of (7) is

$$
\begin{gathered}
\left(\delta \alpha^{-1}-\delta\right) \mathrm{xyz}=0 . \\
\text { i.e., } \delta\left(\alpha^{-1}-1\right) \mathrm{xyz}=0 .
\end{gathered}
$$

Since $\mathrm{xyz} \neq 0$ and $\alpha \neq 1$,we get $\delta=0$.

Hence from (5) we get $\mathrm{xy}^{2}=0$.

Also, if in (7), we choose $\alpha_{3}=0, \alpha_{4}=\alpha_{2}=1$ and $\alpha_{1}=-\beta$, the right hand side of (7) is zero whereas the left hand side of (7) is

$$
\begin{gathered}
\left(-\beta+\beta \alpha^{-1}\right) \mathrm{xyz}=0 \\
\text { i.e., } \beta\left(\alpha^{-1}-1\right) \mathrm{xyz}=0 .
\end{gathered}
$$

Since $x y z \neq 0$ and $\alpha \neq 1$, we get $\beta=0$.

Hence from (4), we get $\mathrm{xz}^{2}=0$.

Then (6) becomes

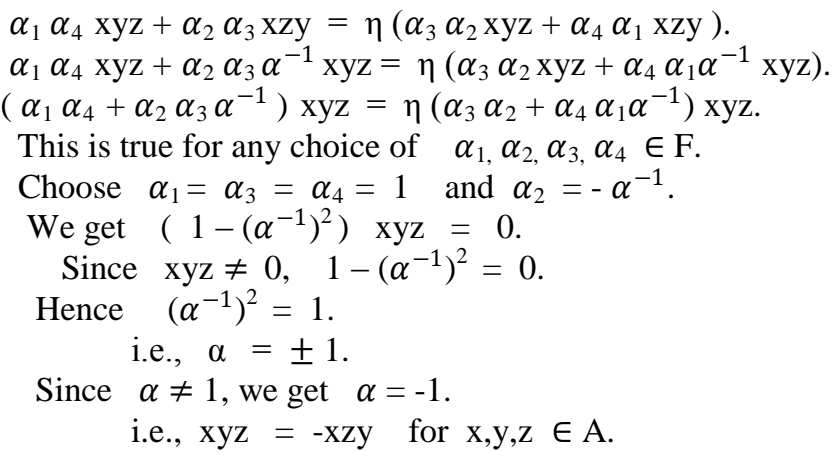

Thus $\mathrm{A}$ is either weak commutative or anti-weak commutative.

\subsection{Lemma:}

Let $\mathrm{A}$ be an algebra( not necessarily associative )over a commutative ring R.Suppose

$\mathrm{A}$ is scalar weak commutative. Then for all $\mathrm{x}, \mathrm{y}, \mathrm{z} \in \mathrm{A}, \alpha \in \mathrm{R}, \alpha \mathrm{xyz}=0$ if and only if $\alpha \mathrm{xzy}=0$.

Also $\mathrm{xyz}=0$ if and only if $\mathrm{xzy}=0$. 


\section{Proof:}

Let $\mathrm{x}, \mathrm{y}, \mathrm{z} \in \mathrm{A}$ and $\alpha \in \mathrm{R}$ such that $\alpha \mathrm{xyz}=0$.

Since $\mathrm{A}$ is scalar weak commutative,there exists $\beta=\beta(\alpha \mathrm{x}, \mathrm{z}, \mathrm{y}) \in \mathrm{R}$ such that $\alpha \mathrm{xzy}=\beta \alpha \mathrm{xyz}=0$.

Similarly if $\alpha$ xzy $=0$, then there exists $\gamma=\gamma(\alpha \mathrm{x}, \mathrm{y}, \mathrm{z}) \in \mathrm{R}$ such that

Thus $\alpha \mathrm{xyz}=0$ iff $\alpha \mathrm{xzy}=0$.

$$
\alpha \mathrm{xyz}=\gamma \alpha \mathrm{xzy}=0 \text {. }
$$

Assume $\mathrm{xyz}=0$. Since $\mathrm{A}$ is scalar commutative, there exists $\delta=\delta(\mathrm{x}, \mathrm{y}, \mathrm{z}) \in \mathrm{R}$ such that $\mathrm{xzy}=\delta \mathrm{xyz}=0$.

Similarly if $\mathrm{xzy}=0$, there exists $\eta=\eta(\mathrm{x}, \mathrm{y}, \mathrm{z}) \in \mathrm{R}$ such that $\mathrm{xyz}=\eta \mathrm{xzy}=0$.

Thus $\mathrm{xyz}=0$ if and only if $\mathrm{xzy}=0$.

\subsection{Lemma:}

Let $\mathrm{A}$ be an algebra over a commutative ring R.Suppose $\mathrm{A}$ is scalar weak commutative.

Let $\mathrm{x}, \mathrm{y}, \mathrm{z}, \mathrm{u} \in \mathrm{A}, \alpha, \beta \in \mathrm{R}$ such that $\mathrm{zu}=\mathrm{uz}, \mathrm{xzy}=\alpha \mathrm{xyz}$ and $\mathrm{x}(\mathrm{y}+\mathrm{u}) \mathrm{z}=\beta \mathrm{xz}(\mathrm{y}+\mathrm{u})$.

Then $\mathrm{x}(\mathrm{zu}-\alpha \mathrm{zu}-\beta \mathrm{zu}+\alpha \beta \mathrm{zu})=0$.

Proof:

From (1) we get

$$
\begin{array}{rr}
\mathrm{x}(\mathrm{y}+\mathrm{u}) \mathrm{z}=\beta \mathrm{xz}(\mathrm{y}+\mathrm{u}) & \rightarrow(1) \\
\mathrm{xzy}=\alpha \mathrm{xyz} & \rightarrow(2) \\
\text { and } \mathrm{zu}=\mathrm{uz} & \rightarrow(3)
\end{array}
$$

$$
\begin{gathered}
\mathrm{xyz}+\mathrm{xuz}=\beta \mathrm{xzy}+\beta \mathrm{xzu} . \\
\mathrm{xyz}+\mathrm{xuz}=\beta \alpha \mathrm{xzy}+\beta \mathrm{xzu} . \\
\mathrm{x}\{\mathrm{yz}+\mathrm{uz}-\alpha \beta y z-\beta \mathrm{zu}\}=0 . \\
\mathrm{x}\{\mathrm{yz}+\mathrm{uz}-\alpha \beta y z-\beta \mathrm{uz}\}=0 . \\
\mathrm{x}(\mathrm{y}+\mathrm{u}-\alpha \beta y-\beta \mathrm{u}) \mathrm{z}=0 .
\end{gathered}
$$

By Lemma 3.3 we get

$$
\begin{array}{r}
\mathrm{xz}(\mathrm{y}+\mathrm{u}-\alpha \beta y-\beta \mathrm{u})=0 . \\
\text { i.e., } \mathrm{xzy}+\mathrm{xzu}-\alpha \beta x y z-\beta \mathrm{xzu}=0 .
\end{array}
$$

i.e., $\alpha \mathrm{xyz}+\mathrm{xzu}-\alpha \beta x y z-\beta \mathrm{xzu}=0$.

Now from (1) we get

$$
\mathrm{xyz}-\beta \mathrm{xzy}=\beta \mathrm{xzu}-\mathrm{xuz} .
$$

$$
\mathrm{xyz}+\mathrm{xuz}=\beta \mathrm{xzy}+\beta \mathrm{xzu} .
$$

Multiplying by $\alpha$ we get,

$$
\alpha \mathrm{xyz}-\alpha \beta \mathrm{xzy}=\alpha \beta \mathrm{xzu}-\alpha \mathrm{xuz} \text {. }
$$

From (4) and (5) we vget

$$
\mathrm{xzu}-\beta \mathrm{xzu}+\alpha \beta \mathrm{xzu}-\alpha \mathrm{xuz}=0 .
$$

i.e., $\mathrm{x}\{\mathrm{zu}-\beta \mathrm{zu}+\alpha \beta \mathrm{zu}-\alpha \mathrm{uz}\}=0$

$$
\mathrm{x}\{\mathrm{zu}-\alpha \mathrm{zu}-\beta \mathrm{zu}+\alpha \beta \mathrm{uz}\}=0 \text {. }
$$

\subsection{Corollary:}

$$
\begin{aligned}
& \text { Taking } \mathrm{u}=\mathrm{z} \text {,we get } \\
& \quad \mathrm{x}\left\{\mathrm{z}^{2}-\alpha \mathrm{z}^{2}-\beta \mathrm{z}^{2}+\alpha \beta \mathrm{z}^{2}\right\}=0 \\
& \text { i.e., } \mathrm{x}(\mathrm{z}(\mathrm{z}-\alpha \mathrm{z})-\beta \mathrm{z}(\mathrm{z}-\alpha \mathrm{z}))=0 \\
& \text { i.e., } \mathrm{x}(\mathrm{z}-\alpha \mathrm{z})(\mathrm{z}-\beta \mathrm{z})=0 \text {. }
\end{aligned}
$$

\subsection{Theorem:}

Let $\mathrm{A}$ be an algebra over a commutative ring R.Suppose $\mathrm{A}$ has no zero divisors.If $\mathrm{A}$ is scalar weak commutative, then $\mathrm{A}$ is weak commutative.

\section{Proof:}

Let $\mathrm{x}, \mathrm{y}, \mathrm{z} \in \mathrm{A}$. Since $\mathrm{A}$ is scalar weak commutative, there exist scalars $\alpha=\alpha(\mathrm{x}, \mathrm{z}, \mathrm{y}) \in \mathrm{R}$ and $\beta=\beta(\mathrm{x}, \mathrm{y}+\mathrm{z}, \mathrm{z}) \in R$ such that

and $\mathrm{x}(\mathrm{y}+\mathrm{z}) \mathrm{z}=\beta \mathrm{xz}(\mathrm{y}+\mathrm{z})$

$$
\mathrm{xzy}=\alpha \mathrm{xy} \quad \rightarrow(1)
$$$$
\rightarrow(2)
$$

Then by the above corollary, we get

$$
\mathrm{x}(\mathrm{z}-\alpha \mathrm{z})(\mathrm{z}-\beta \mathrm{z})=0 .
$$

Since A has no zero divisors

$$
\mathrm{z}=\alpha \mathrm{z} \text { or } \mathrm{z}=\beta \mathrm{z} \text {. }
$$

If $\mathrm{z}=\alpha \mathrm{z}$, then from (1) we get 
$\mathrm{xzy}=\mathrm{xyz}$

If $\mathrm{z}=\beta \mathrm{z}$, then from (2) we get

$\mathrm{x}(\mathrm{y}+\mathrm{z}) \mathrm{z}=\mathrm{x} \mathrm{z}(\mathrm{y}+\mathrm{z})$

$x y z+x z^{2}=x z y+x z^{2}$

i.e., $x y z=x z y$.

Thus $\mathrm{A}$ is weak commutative.

\subsection{Definition:}

Let $\mathrm{R}$ be any ring and $\mathrm{x}, \mathrm{y}, \mathrm{z} \in \mathrm{R}$.We define $\mathrm{xyz}-\mathrm{xzy}$ as the weak commutator of $\mathrm{x}, \mathrm{y}, \mathrm{z}$ .i.e., $x y z-x z y=x[y, z]$ is called the weak commutator of $x, y, z$.

\subsection{Theorem:}

Let $\mathrm{A}$ be an algebra over a commutative ring R.Let $\mathrm{A}$ be scalar weak commutative.If $\mathrm{A}$ has an identity,then the square of every weak commutator is zero.

Proof:

$$
\text { i.e., }(x y z-x z y)^{2}=0 \text { for all } x, y, z \in A \text {. }
$$

Let $\mathrm{x}, \mathrm{y}, \mathrm{z} \in \mathrm{A}$.Since $\mathrm{A}$ is scalar weak commutative, there exist scalars $\alpha=\alpha(\mathrm{x}, \mathrm{y}, \mathrm{z}) \in \mathrm{R}$ and $\beta=\beta(\mathrm{x}, \mathrm{y}+1, \mathrm{z}) \in \mathrm{R}$ such that

$$
\mathrm{x}(\mathrm{y}+1) \mathrm{z}=\beta \mathrm{xz}(\mathrm{y}+1) \quad \rightarrow(2)
$$

From (2) we get

$$
\begin{aligned}
& \mathrm{xyz}+\mathrm{xz}-\beta \mathrm{xzy}-\beta \mathrm{xz}=0 \\
& \mathrm{xyz}+\mathrm{xz}-\beta \alpha \mathrm{xyz}-\beta \mathrm{xz}=0 \\
& \mathrm{xyz}+\mathrm{xz}-\alpha \beta \mathrm{xyz}-\beta \mathrm{xz}=0
\end{aligned}
$$

i.e., $\mathrm{x}(\mathrm{y}+1-\alpha \beta \mathrm{y}-\beta) \mathrm{z}=0$

Using Lemma 3.3 we get

$$
\begin{aligned}
& \mathrm{xz}(\mathrm{y}+1-\alpha \beta \mathrm{y}-\beta) \mathrm{z}=0 \\
& \mathrm{xz} \mathrm{y}+\mathrm{xz}-\alpha \beta \mathrm{xzy}-\beta \mathrm{xz}=0 \\
& \alpha \mathrm{x} \mathrm{yz}+\mathrm{xz}-\alpha \beta \mathrm{xzy}-\beta \mathrm{xz}=0
\end{aligned}
$$

Also from (2) we get

$$
\mathrm{xyz}+\mathrm{xz}=\beta \mathrm{xzy}+\beta \mathrm{xz}
$$

Multiplying by $\alpha$ we get

$$
\alpha \mathrm{xyz}+\alpha \mathrm{xz}=\alpha \beta \mathrm{xzy}+\alpha \beta \mathrm{xz}
$$

i.e., $\alpha \mathrm{xyz}-\alpha \beta \mathrm{xzy}=\alpha \beta \mathrm{xz}-\alpha \mathrm{xz}$.

From (3) and (4) we get

$$
\mathrm{xz}-\beta \mathrm{xz}+\alpha \beta \mathrm{xz}-\alpha \mathrm{xz}=0 .
$$

i.e., $\quad \mathrm{xz}-\alpha \mathrm{xz}-\beta \mathrm{xz}+\alpha \beta \mathrm{xz}=0$.

i.e., $\mathrm{x}(\mathrm{z}-\alpha \mathrm{z})=\mathrm{x}(\beta \mathrm{z}-\alpha \beta \mathrm{z})$

Multiplying by $y+1$ on the right we get

$$
\begin{aligned}
\mathrm{x}\{\mathrm{z}(\mathrm{y}+1)-\alpha \mathrm{z}(\mathrm{y}+1)\} & =\mathrm{x}\{\beta \mathrm{z}(\mathrm{y}+1)-\alpha \beta \mathrm{z}(\mathrm{y}+1)\} \\
& =\beta \mathrm{xz}(\mathrm{y}+1)-\alpha \beta \mathrm{xz}(\mathrm{y}+1) \\
& =\mathrm{x}(\mathrm{y}+1) \mathrm{z}-\alpha \mathrm{x}(\mathrm{y}+1) \mathrm{z} \\
& =\mathrm{x}\{(\mathrm{y}+1) \mathrm{z}-\alpha(\mathrm{y}+1) \mathrm{z}\}
\end{aligned} \quad(\mathrm{using}(2))
$$

i.e., $x\{z(y+1)-\alpha z(y+1)\}=x\{(y+1) z-\alpha(y+1) z\}$

i.e., $\mathrm{x}\{\mathrm{z}(\mathrm{y}+1)-(\mathrm{y}+1) \mathrm{z}\}=\mathrm{x}\{\alpha \mathrm{z}(\mathrm{y}+1)-\alpha(\mathrm{y}+1) \mathrm{z}\}$

i.e., $\mathrm{x}\{\mathrm{zy}+\mathrm{z}-\mathrm{yz}-\mathrm{z}\}=\alpha \mathrm{x}\{\mathrm{zy}+\mathrm{z}-\mathrm{yz}-\mathrm{z}\}$

$$
\mathrm{x}\{\mathrm{zy}-\mathrm{yz}\}=\alpha \mathrm{x}\{\mathrm{zy}-\mathrm{yz}\}
$$

i.e., $\mathrm{x}\{\mathrm{zy}-\alpha \mathrm{zy}\}=\mathrm{x}\{\mathrm{yz}-\alpha \mathrm{yz}\}$

i.e., $\mathrm{xyz}-\alpha \mathrm{xyz}=\mathrm{xzy}-\alpha \mathrm{xzy}$

i.e., $\mathrm{xyz}-2 \alpha \mathrm{xyz}+\alpha^{2} \mathrm{xyz}=0$

$$
=\alpha \mathrm{xyz}-\alpha \alpha \mathrm{xyz}
$$

i.e., $\mathrm{x}\left(\mathrm{y}-2 \alpha \mathrm{y}+\alpha^{2} \mathrm{y}\right) \mathrm{z}=0$

Now, $(\mathrm{xyz}-\mathrm{xzy})^{2}=(\mathrm{xyz}-\alpha \mathrm{xyz})^{2} \quad($ using $(1))$

$$
\begin{aligned}
= & (\mathrm{xyz}-\alpha \mathrm{xyz})(\mathrm{xyz}-\alpha \mathrm{xyz}) \\
& =\mathrm{xyz} \mathrm{xyz}-\alpha \mathrm{xyz} \mathrm{xyz}-\alpha \mathrm{xyz} \mathrm{xyz}+\alpha^{2} \mathrm{xyz} \mathrm{xyz} \\
& =\mathrm{xyz} \mathrm{xyz}-2 \alpha \mathrm{xyz} \mathrm{xyz}+\alpha^{2} \mathrm{xyz} \mathrm{xyz}
\end{aligned}
$$$$
=\mathrm{x}\left(\mathrm{y}-2 \alpha \mathrm{y}+\alpha^{2} \mathrm{y}\right) \mathrm{zxyz}
$$$$
=0 \text {. }
$$

$$
=0 . \mathrm{xyz} \quad(\operatorname{using}(5))
$$

Thus $(x y z-x z y)^{2}=0$. 
i.e., Square of every weak commutator is zero.

\subsection{Definition:}

Let $\mathrm{R}$ be a P.I.D ( principal ideal domain ) and $\mathrm{A}$ be an algebra over R.Let a $\in \mathrm{A}$.

Then the order of a,denoted an $\mathrm{O}$ (a) is defined to be the generator of the ideal $\mathrm{I}=\{\alpha \in \mathrm{R} \mid \alpha \mathrm{a}=0\}$.

$\mathrm{O}(\mathrm{a})$ is unique upto associates and $\mathrm{O}(\mathrm{a})=1$ if and only if $\mathrm{a}=0$.

\subsection{Lemma:}

Let $\mathrm{A}$ be an algebra with unity over a principal ideal domain R.If A is scalar weak commutative,

$\mathrm{z} \in \mathrm{A}$ such that $\mathrm{O}(\mathrm{z})=0$, then $\mathrm{xyz}=\mathrm{xzy}$ for all $\mathrm{x}, \mathrm{y}, \mathrm{z} \in \mathrm{A}$.

Proof:

Let $\mathrm{z} \in \mathrm{A}$ with $\mathrm{O}(\mathrm{z})=0$.

For $\mathrm{x}, \mathrm{y} \in \mathrm{A}$,there exists scalars $\alpha=\alpha(\mathrm{x}, \mathrm{y}, \mathrm{z}) \in \mathrm{R}$ and $\beta=\beta(\mathrm{x}, \mathrm{y}+1, \mathrm{z}) \in \mathrm{R}$ such that

From (2) we get

$$
\begin{array}{ll}
\mathrm{xzy}=\alpha \mathrm{xyz} & \rightarrow(1) \\
\mathrm{x}(\mathrm{y}+1) \mathrm{z}=\beta \mathrm{xz}(\mathrm{y}+1) & \rightarrow(2)
\end{array}
$$

$$
\begin{aligned}
& \mathrm{xyz}+\mathrm{xz}-\beta \mathrm{xzy}-\beta \mathrm{xz}=0 \\
& \mathrm{xyz}+\mathrm{xz}-\alpha \beta \mathrm{xyz}-\beta \mathrm{xz}=0 \\
& \mathrm{x}(\mathrm{y}+1-\alpha \beta \mathrm{y}-\beta .1) \mathrm{z}=0
\end{aligned}
$$

Using Lemma 3.3 we get

$$
\begin{aligned}
& \mathrm{xz}(\mathrm{y}+1-\alpha \beta \mathrm{y}-\beta .1)=0 \\
& \mathrm{xzy}+\mathrm{xz}-\alpha \beta \mathrm{xzy}-\beta \mathrm{xz})=0 \\
& \alpha \mathrm{xzy}+\mathrm{xz}-\alpha \beta \mathrm{xzy}-\beta \mathrm{xz}=0
\end{aligned}
$$

From (2) we get

$$
\mathrm{xyz}+\mathrm{xz}=\beta \mathrm{xzy}+\beta \mathrm{xz}
$$

Multiplying by $\alpha$ we get

$$
\alpha \mathrm{xyz}+\alpha \mathrm{xz}=\alpha \beta \mathrm{xzy}+\alpha \beta \mathrm{xz}
$$

i.e., $\alpha \mathrm{xyz}-\alpha \beta \mathrm{xzy}=\alpha \beta \mathrm{xz}-\alpha \mathrm{xz}$

From (3) and (4) we get

$$
\mathrm{xz}-\beta \mathrm{xz}+-\alpha \beta \mathrm{xz}-\alpha \mathrm{xz}=0
$$

$(1-\alpha)(1-\beta) \mathrm{xz}=0 \quad \forall \mathrm{x} \in \mathrm{A}$.

Then there exist scalars $\gamma \in \mathrm{R}, \delta \in \mathrm{R}$ such that $\gamma \mathrm{xz}=0$

and

From (7)

$$
\delta(\mathrm{x}+1) \mathrm{z}=0 \quad \rightarrow(7)
$$

$$
\delta \mathrm{xz}+\delta \mathrm{z}=0
$$

Multiply by $\gamma$

$$
\begin{array}{ll}
\gamma \delta \mathrm{xz}+\gamma \delta \mathrm{z}=0 & \rightarrow(8) \\
\gamma \delta \mathrm{xz}=0 & \rightarrow(9)
\end{array}
$$

From (6) we get

From (8) and (9) we get

$$
\gamma \delta \mathrm{z}=0
$$

Since $\mathrm{O}(\mathrm{z})=0$ we get $\gamma=0$ and $\delta=0$.

Then from $1-\alpha=0$ or $1-\beta$.

If $\alpha=1$,from (1) we get $\mathrm{xzy}=\mathrm{xyz}$.

If $\beta=1$,from (2) we get

$$
\begin{aligned}
& x(y+1) z=x z(y+1) \\
& x y z+x z=x z y+x z \\
& x y z=x z y
\end{aligned}
$$

\subsection{0 (a) Lemma:}

Let A be an algebra with idemtity over Principal ideal domain R.If A is scalar weak commutative, $y \in R$ with $O(y)=0$, then $y$ is in the center of $A$.

Proof:

Let $\mathrm{y} \in \mathrm{A}$ with $\mathrm{O}(\mathrm{y})=0$.

For any $\mathrm{x} \in \mathrm{A}$,there exist scalars $\alpha=\alpha(1, \mathrm{x}, \mathrm{y}) \in \mathrm{R}$ and $\beta=\beta(1, \mathrm{y}, \mathrm{x}+1) \in \mathrm{R}$ such that

(i.e) $1 . \mathrm{xy}=\alpha \cdot 1 . \mathrm{yx}$.

$$
\mathrm{xy}=\alpha \mathrm{yx}
$$


and 1. $\mathrm{y}(\mathrm{x}+1)=\beta$.1. $(\mathrm{x}+1) \mathrm{y}$

(i.e)., $\mathrm{y}(\mathrm{x}+1)=\beta(\mathrm{x}+1) \mathrm{y}$

From (2) we get

$$
\begin{aligned}
& \mathrm{yx}+\mathrm{y}=\beta \mathrm{xy}+\beta \mathrm{y} \\
& \mathrm{yx}+\mathrm{y}=\alpha \beta \mathrm{xy}+\beta \mathrm{y} \\
& \mathrm{yx}+\mathrm{y}-\alpha \beta \mathrm{xy}-\beta \mathrm{y}=0 . \\
& 1 . \mathrm{y}(\mathrm{x}+1-\alpha \beta \mathrm{x}-\beta .1)=0 .
\end{aligned}
$$

By Lemma 3.3

$$
\begin{aligned}
& \text { 1. } \quad(\mathrm{x}+1-\alpha \beta \mathrm{x}-\beta .1) \mathrm{y}=0 \\
& \mathrm{xy}+\mathrm{y}-\alpha \beta \mathrm{xy}-\beta \mathrm{y}=0
\end{aligned}
$$

Also from (2)

Multiply by $\alpha$

$$
\mathrm{yx}+\mathrm{y}-\beta \mathrm{xy}-\beta \mathrm{y}=0 \text {. }
$$

$$
\begin{aligned}
& \alpha \mathrm{yx}+\alpha \mathrm{y}-\alpha \beta \mathrm{xy}-\alpha \beta \mathrm{y}=0 \\
& \mathrm{xy}+\alpha \mathrm{y}-\alpha \beta \mathrm{xy}-\alpha \beta \mathrm{y}=0
\end{aligned}
$$

From (3) and (4) we get

$$
\begin{aligned}
& \mathrm{y}-\beta \mathrm{y}-\alpha \mathrm{y}+\alpha \beta \mathrm{y}=0 \\
& (\mathrm{y}-\beta \mathrm{y})-\alpha(\mathrm{y}-\beta \mathrm{y})=0 \\
& (1-\alpha)(\mathrm{y}-\beta \mathrm{y})=0 \\
& (1-\alpha)(1-\beta) \mathrm{y}=0
\end{aligned}
$$

Since $\mathrm{O}(\mathrm{y})=0$, we get $\alpha=1$ or $\beta=1$.

If $\alpha=1$, from (1) we get $\mathrm{xy}=\mathrm{yx}$.

If $\beta=2$, from (2) we get

$$
y(x+1)=(x+1) y
$$

i.e., $y x+y=x y+y$

$$
\mathrm{yx}=\mathrm{xy}
$$

i.e., $y$ commutes with $x$.

As $x \in A$ is arbitrary, $y$ is in the center.

\subsection{Lemma:}

Let $\mathrm{A}$ be an algebra with identity over a P.I.D R.Suppose that $\mathrm{A}$ is scalar weak commutative.

Assume further that there exists a prime $\mathrm{p} \in \mathrm{R}$ and positive integer $\mathrm{m} \in z^{+}$such that $\mathrm{p}^{\mathrm{m}} \mathrm{A}=0$. Then $\mathrm{A}$ is Weak commutative.

\section{Proof:}

Let $\mathrm{O}(\mathrm{xy})=\mathrm{p}^{\mathrm{k}}$ for some $\mathrm{k} \in Z^{+}$.

We prove by induction on $\mathrm{k}$ that $\mathrm{uxy}=$ uyx for all $\mathrm{u} \in \mathrm{A}$.

If $\mathrm{k}=0$, then $\mathrm{O}(\mathrm{xy})=\mathrm{p}^{0}=1$ and so $\mathrm{xy}=0$.

So uxy $=0$.Also by Lemma 3.3 uyx $=0$.

Hence $u x y=u y x$ for all $\mathrm{u} \in$ A.So,assume that $\mathrm{k}>0$ and that the statement is true for $1>\mathrm{k}$.

We first prove that for any $u \in A, u x y-u y x \neq 0$ implies $\omega$ (uy) $x-\omega x$ (uy) $=0$ for all $\omega \in A$.

So, let uxy - uyx $\neq 0$.

Since A is scalar weak commutative, there exist scalars $\alpha=\alpha(\mathrm{u}, \mathrm{x}, \mathrm{y}) \in \mathrm{R}$ and $\beta=\beta(\mathrm{u}, \mathrm{x}+1, \mathrm{y}) \in \mathrm{R}$ such that
and
$\mathrm{uxy}=\alpha$ uyx
$\mathrm{u}(\mathrm{x}+1) \mathrm{y}=\beta$ uy $(\mathrm{x}+1)$

From (2) we get

$$
\mathrm{uxy}+\mathrm{uy}=\beta \mathrm{uyx}+\beta \mathrm{uy}
$$

$\alpha$ uyx + uy $=\beta$ uyx $+\beta$ uy

(using (1))

$(\alpha-)$ uyx $=(\beta-1)$ uy

If $(\alpha-\beta)$ uyx $=0$ then $(\beta-1)$ uy $=0$ and so $\beta$ uy $=$ uy.So from (2) we get

$$
u(x+1) y=u y(x+1)
$$

i.e., $u x y+$ uy $=$ uyx + uy.

So

i.e., uxy - uyx $=0$, contradicting our assumption that uxy - uyx $\neq 0$.

$(-\beta)$ uyx $\neq 0$.In particular $\alpha-\beta \neq 0$.

Let $\alpha-\beta=\mathrm{p}^{\mathrm{t}} \delta$ for some $\mathrm{t} \in \mathrm{Z}^{+}$and $\delta \in \mathrm{R}$ with $(\delta, \mathrm{p})=1$.If $\mathrm{t} \geq \mathrm{k}$, then since $\mathrm{O}(\mathrm{xy})=\mathrm{p}^{\mathrm{k}}$, we would 
get $(\alpha-\beta)$ uxy $=0$, a contradiction. Hence $\mathrm{t}<\mathrm{k}$.

Now, since $\mathrm{p}^{\mathrm{k}} \mathrm{uxy}=0$, by Lemma 3.3, we have

So from (3), $\mathrm{p}^{\mathrm{k}-\mathrm{t}}(\beta-1)$ uy $=\mathrm{p}^{\mathrm{k}-\mathrm{t}}(\alpha-\beta)$ uyx

$$
\mathrm{p}^{\mathrm{k}} \operatorname{uyx}=0 .
$$

Let $\mathrm{O}($ uy $)=\mathrm{p}^{\mathrm{i}}$ for some $\mathrm{i} \in \mathrm{Z}^{+}$.

$$
\begin{aligned}
& =\mathrm{p}^{\mathrm{k}-\mathrm{t}} \mathrm{p}^{\mathrm{t}} \delta \text { uyx. } \\
& =\mathrm{p}^{\mathrm{k}} \delta \text { uyx }=0 .
\end{aligned}
$$

If $\mathrm{i}<\mathrm{k}$ then by induction hypothesis $\mathrm{uxy}=\mathrm{uyx}$, contradiction to our assumption that $\mathrm{uxy}-\mathrm{uyx} \neq 0$.

So $\mathrm{i} \geq \mathrm{k}$.

Hence

$$
\mathrm{P}^{\mathrm{k}}\left|\mathrm{P}^{\mathrm{i}}\right| \mathrm{p}^{\mathrm{k}-\mathrm{t}}(\beta-1) \text {. }
$$

Thus $\mathrm{p}^{\mathrm{t}} \mid \beta-1$ and let $\beta-1=\mathrm{p}^{\mathrm{t}} \gamma$ for some $\gamma \in \mathrm{R}$.

From (3) we get $(\alpha-\beta)$ uyx $=(\beta-1)$ uy.

$$
\mathrm{p}^{\mathrm{t}} \delta \text { uyx }=\mathrm{p}^{\mathrm{t}} \gamma \text { uy } \quad \text { (using (4) and (5)) }
$$

i.e., $\mathrm{p}^{\mathrm{t}}(($ uy) $(\delta \mathrm{x}-\gamma .1))=0$. Hence by induction hypothesis

$\omega($ uy $)(\delta \mathrm{x}-\gamma .1) \quad=\omega(\delta \mathrm{x}-\gamma .1)$ (uy) $\quad \forall \omega \in \mathrm{A}$

$\omega$ ( uy ) $\delta \mathrm{x}-\omega$ (uy ) $\gamma .1=\omega \delta \mathrm{x}$ (uy) $-\omega \gamma .1$ (uy)

i.e., $\omega$ (uy) $\delta \mathrm{x}-\gamma \cdot \omega$ (uy) $\quad=\omega \delta \mathrm{x}$ (uy) $-\gamma \omega$ (uy)

$\delta\{$ ( uy ) $\mathrm{x}-\omega \mathrm{x}$ (uy) $\}=0$

Since $(\delta, \mathrm{p})=1$, there exist,$\gamma \in \mathrm{R}$ such that $\mu \mathrm{p}^{\mathrm{m}}+\gamma \delta=1$.

$\therefore \mu \mathrm{p}^{\mathrm{m}}\{\omega$ ( uy ) $\mathrm{x}-\omega \mathrm{x}$ (uy) $\}+\gamma \delta\{\omega$ ( uy ) $\mathrm{x}-\omega \mathrm{x}$ (uy) $\}$

$$
0+0 \quad=\omega(\text { uy }) \mathrm{x}-\omega \mathrm{x} \text { (uy) } \quad\left(\because \mathrm{p}^{\mathrm{m}} \mathrm{A}=0\right)
$$

i.e., $\quad \omega$ (uy ) $\mathrm{x}=\omega \mathrm{x}$ (uy )

i.e., uyx $\neq$ uxy implies $\omega$ (uy ) $\mathrm{x}=\omega \mathrm{x}$ (uy ) for all $\omega \in \mathrm{A} \quad \rightarrow(7)$

Now, we proceed to show that uxy = uyx for all $u \in A$.

Suppose not there exist $\mathrm{u} \in \mathrm{A}$ such that uyx $\neq \mathrm{uxy}$

Then we also have $(\mathrm{u}+1) \mathrm{yx} \neq(\mathrm{u}+1) \mathrm{xy}$

From (7) and (8) we get

$$
\begin{aligned}
& \omega(\mathrm{uy}) \mathrm{x}=\omega \mathrm{x}(\mathrm{uy}) \text { for all } \omega \in \mathrm{A} \\
& \omega(\mathrm{u}+1) \mathrm{yx} \neq(\mathrm{u}+1) \mathrm{xy} \text { for all } \omega \in \mathrm{A}
\end{aligned}
$$

From (11) we get $\omega$ (uy ) $\mathrm{x}+\omega \mathrm{yx}=\omega \mathrm{x}($ uy $)+\omega \mathrm{xy}$ for all $\omega \in \mathrm{A}$.

i.e., $\omega \mathrm{yx}=\omega \mathrm{xy}$ for all $\omega \in \mathrm{A}$ ( using (10)) a contradiction.

This contradiction prove that uxy = uyx for all $u \in A$.

Thus A is vweak commutative.

\subsection{Lemma:}

Let $\mathrm{A}$ be an algebra with identity over a principal ideal domain R.If $\mathrm{A}$ is scalar weak commutative,then $\mathrm{A}$ is weak commutative.

\section{Proof:}

Suppose $\mathrm{A}$ is not weak commutative, there exists $\mathrm{z} \in \mathrm{A}$ such that $\mathrm{xyz} \neq \mathrm{xzy}$ for all $\mathrm{x}, \mathrm{y} \in \mathrm{A}$.

Also $\mathrm{xy}(\mathrm{z}+1) \neq \mathrm{x}(\mathrm{z}+1) \mathrm{y}$.

Hence by Lemma 3.9, $\mathrm{O}(\mathrm{z}) \neq 0$ and $\mathrm{O}(\mathrm{z}+1) \neq 0$.

Hence $\mathrm{O}(1) \neq 0$. Let $\mathrm{O}(1)=\mathrm{d} \neq 0$. Then $\mathrm{d}$ is not a unit and hence $\mathrm{d}=p_{1}^{i_{1}} p_{2}^{i_{2}} p_{3}^{i_{3}}$

Some primes $\mathrm{p}_{1}, \mathrm{p}_{2}, \mathrm{p}_{3}$.... $\mathrm{P}_{\mathrm{k}} \in \mathrm{A}$ some positive integers $\mathrm{i}_{1}, \mathrm{i}_{2}, \ldots \ldots \ldots \ldots \ldots \mathrm{i}_{\mathrm{k}}$. $p_{k}^{i_{k}}$ for

Let $\mathrm{A}_{\mathrm{j}}=\left\{\mathrm{a} \in \mathrm{A} \mid p_{j}^{i_{j a}}=0\right\}$.Then each $\mathrm{A}_{\mathrm{j}}$ is a non zero subalgebra of $\mathrm{A}$ and $A=A_{1} \oplus A_{2} \ldots \ldots \ldots \ldots \oplus A_{k}$.

Being subalgebras of $A$,each $A_{i}$ is scalar weak commutative.Being homomorphic image of $A$,all the Ai' s have identity elements.By Lemma 3.10 each $\mathrm{A}_{\mathrm{i}}$ is weak commutative and hence $\mathrm{A}$ is weak commutative, a contradiction.Then contradiction proves that $\mathrm{A}$ is weak commutative. 


\section{References}

[1]. R.Coughlin and M.Rich, On Scalar dependent algebras, Canada J.Math, 24(1972), 696- 702.

[2]. R.Coughlin and K.Kleinfield and M.Rich, Scalars dependent algebras, Proc.Amer.Math.Soc, 39 (1973), 69 - 73.

[3]. G.Gopalakrishnamoorthy, S.Geetha and S.Anitha, On Quasi - weak m-power Commutative Near-rings and Quasi - weak ( m,n ) power commutativenear - rings, IOSR Jour.of.Math, vol 12(4), (2016), 87-90.

[4]. G.Gopalakrishnamoorthy, S.Geetha and S.Anitha, On Quasi-weak Commutative Boolean-like near-rings, Malaya Journal of Mathematik, 3(3), (2015), $318-326$.

[5]. G.Gopalakrishnamoorthy, S.Geetha and S.Anitha, On Weak m power Commutative Near-ring and Weak ( m,n ) power commutative near- rings.

[6]. K.Koh,J.Luh and M.S.Putcha, On the associativity and commutativity of algebras over Commutative rings, Porcific.Journal of Math, 63, No. 2 ,(1976), 423 - 430.

[7]. Pliz, Glinter, Near - rings, North Holland, Aneter dam, (1983)

[8]. M.Rich, A Commutativity theorem for algebras, Amer, Math, Monthly, 82 (1975), 377 - 379. 